\title{
Rough Sets and Fuzzy Sets Theory Applied to the Sequential Medical Diagnosis
}

\author{
Andrzej Zolnierek and Marek Kurzynski \\ Wroclaw University of Technology, Faculty of Electronics, Chair of Systems and \\ Computer Networks, Wyb. Wyspianskiego 27, 50-370 Wroclaw, Poland \\ \{andrzej.zolnierek, marek. kurzynski\}@pwr.wroc.pl
}

\begin{abstract}
Sequential classification task is typical in medical diagnosis, when the investigations of the patient's state are repeated several times. Such situation always takes place in the controlling of the drug therapy efficacy. A specific feature of this diagnosis task is the dependence between patient's states at particular instants, which should be taken into account in sequential diagnosis algorithms. In this paper methods for performing sequential diagnosis using fuzzy sets and rough sets theory are developed and evaluated. For both soft methodologies several algorithms are proposed which differ in kind of input data and in details of classification procedures for particular instants of decision process. Proposed algorithms were practically applied to the computer-aided medical problem of recognition of patient's acid-base equilibrium states. Results of comparative experimental analysis of investigated algorithms in respect of classification accuracy are also presented and discussed.
\end{abstract}

\section{Introduction}

In many pattern recognition tasks there exist dependencies among the patterns to be recognized. Such a task, henceforth called the sequential classification (SC) task, involves dealing with a complex decision problem in which the sequences of patterns should be recognized. For instance, in the medical diagnosis we have to deal with such problems in which the patient's state at a given time depends on the preceding states. Although there remains no doubt about existence of this dependence, it may be of a different nature and range; its simplest instance can be a one-instant-backwards dependence to so complex arrangements as those in which the current state depends on the whole former course of the disease including the sequence of applied treatment as well. From the theoretical point of view, during construction of an appropriate decision algorithm we must not limit our approach only to the current feature vector but we have to consider all the available measurement and observed data instead, as they may contain important information about the recognized patient's state at a given instant. The measurement data can comprise all the features vectors and applied treatments observed so far, thus the amount of data is very large and grows over the time from one instant to another. In such a situation performing of SC task various simplifications and compromises must be made. The dependence can be

J.C. Rajapakse, B. Schmidt, and G. Volkert (Eds.): PRIB 2007, LNBI 4774, pp. 311 322, 2007.

(C) Springer-Verlag Berlin Heidelberg 2007 
included at an as early stage as that of formulating a mathematical model for the SC task, or as late as at the stage of selecting the appropriate input data set in the decision algorithm which otherwise does not differ from the classical recognition task. In this paper the second approach is presented. It can be called data-oriented, while it uses methods developed in the field of computational intelligence such as fuzzy logic, rough sets theory and genetic algorithms. These methods are recently becoming increasingly popular in the pattern recognition as an attractive alternative to statistical approach. They can perform classification from both labeled and unlabeled training sets as well as acquire and explore the human expert knowledge. They have been successfully applied in classical pattern recognition tasks, i.e. without taking into account the context [6], and in the sequential classification task [7], [17]. Although the information about former applied treatment can be useful for physicians but including such information in model of recognition requires simplifying assumptions. In consequence in such case, in respective pattern recognition algorithms with learning the number of unknown parameters is growing. It requires more medical data in learning process, but the number of medical data is as usual limited. Then in this chapter comparative analysis of such methodologies, but without taking into account the treatment process, to the problem of medical sequential diagnosis (classification of states of acid-base balance) is described. Let us stress that there are many possible methods for performing sequential diagnosis, but the aim of this work was to compare approaches using fuzzy sets and rough set theory, because both are data-oriented and do not require any additional assumptions. After preliminaries and problem statement, we present several algorithms of SC, which differ from each other using different kind of input data and using either fuzzy logic or rough sets methodology. All presented algorithms were practically applied to the problem of sequential medical diagnosis and results can be found at the end of this chapter.

\section{Preliminaries and the Problem Statement}

We will treat the sequential classification (SC) task as a discrete controlled dynamical process. The pattern (patient) is at the $n$-th instant in the state $j_{n} \in \mathcal{M}$, where $\mathcal{M}$ is an $m$-element set of possible states numbered with the successive natural numbers, thus

$$
j_{n} \in \mathcal{M}=\{1,2, \ldots, m\} .
$$

Obviously, the notion of instant has no specific temporal meaning here, as its interpretation depends on the character of the medical case under consideration. The actual used measure may be minutes, hours, days or even weeks. The patient's state $j_{n}$ is unknown and does not undergo our direct observation. What we can only observe are the symptoms by which a state manifests itself. We will denote an $d$-dimensional symptom vector measured at the $n$-th instant by $x_{n} \in \mathcal{X}$ (thus $\mathcal{X}$ is the observation space). As already mentioned, the patient's current state depends on the history and thus in the general case the decision 
algorithm must take into account the whole sequence of the preceding symptom vectors $\bar{x}_{n}=\left\{x_{1}, x_{2}, \ldots, x_{n}\right\}$ and the course of treatment process. It must be underlined here that sometimes it may be difficult to include all the available data, especially for bigger $n$. In such cases we have to allow some simplifications (e.g. do not take into account the treatments or take into account only several recent values in the sequence of symptom vectors $\bar{x}_{n}$.

In order to classify such sequences of patient's states we need some more general information to take a valid decision, namely the a priori knowledge concerning the general associations that hold between decisions on the one hand, and the sequence of feature on the other. This knowledge may have multifarious forms and various origins. From now on we assume that it has the form of so called training set, which in the investigated diagnostic task consists of $N$ training sequences (patient's records):

$$
\mathcal{S}=\left\{S_{1}, S_{2}, \ldots, S_{N}\right\}
$$

A single patient's record:

$$
S_{k}=\left(\left(x_{1, k}, j_{1, k}\right),\left(x_{2, k}, j_{2, k}\right), \ldots,\left(x_{L, k}, j_{L, k}\right)\right)
$$

denotes a single dynamic process course that comprises $L$ feature observations instatnts and the patient's states. Analysis of the SC task implies that, when considered in its most general form, the explored decision algorithm should use in the $n$-th instant the whole available observed data i.e. the sequences of all feature vectors $\bar{x}_{n}$ as well as the knowledge included in the training set $\mathcal{S}$. In consequence, the algorithm is of the following form:

$$
i_{n}=\Psi_{n}\left(\mathcal{S}, \bar{x}_{n}\right), n=1,2, \ldots, i_{n}, i_{n} \in \mathcal{M} .
$$

The next chapters describe in depth the construction of the sequential diagnosis algorithms (4) using various concepts based on fuzzy and rough sets theory.

\section{Algorithms of SC Based on Fuzzy Sets Theory}

In this section we will apply the fuzzy sets theory to the construction of SC algorithm (4). Two approaches will be considered:

- Mamdani inference system for fuzzy rules with procedure of generating fuzzy rules from learning set developed for sequential classification,

- application of fuzzy relation defined on Cartesian product of input data and class number set obtained from the learning set as a solution of appropriate optimization problem.

For both concepts corresponding algorithms will be proposed which differ in kind of input data and details of classification procedures for particular instants of decision process. 


\subsection{Fuzzy Method with Mamdani Inference System}

This concept consists in applying the inference engine from a fuzzy rule system to construction decision algorithms for SC task. For all the algorithms presented below we assume the following general form of the $k$-th rule in the system $(k=$ $1,2, \ldots, K)$, which associate an observation vector $a=\left(a^{(1)}, a^{(2)}, \ldots, a^{\left(d_{a}\right)}\right)$ with class numbers:

$$
\text { IF } a^{(1)} i s A_{1, k} A N D \cdots A N D a^{(L)} i s A_{L, k} T H E N B_{k} .
$$

$A_{i, k}$ are fuzzy sets (which membership functions are designated by $\mu_{A_{i, k}}$ ) that correspond to the nature of particular input observations, whereas $B_{k}$ is a discrete fuzzy set defined on the class number set $\mathcal{M}$, with the $\mu_{B_{k}}$ membership function.

The only difference between the algorithms is the form of observation vector a and its relation with features of pattern to be recognized and, what follows, the procedure for rule system (5) derivation from the learning set (2).

As recognition algorithm the Mamdani fuzzy inference system has been applied [1], [16]. In this system we use the minimum t-norm as AND connection in premises, product operation as conjunctive implication interpretation in rules, the maximum t-conorm as aggregation operation, and finally the maximum defuzzification method.

Two methods can be used to obtain collection of fuzzy if-then rules (5) in the construction of fuzzy system:

- from human expert or based on domain knowledge,

- extraction of rules using numerical input-output data.

One of the best known method of rules generating from the given training patterns set (2), is the method proposed by Wang and Mendel [15], which developed for the SC will be applied in the further algorithms.

Algorithm Without Context (Mamdani-0). In this case the SC is considered as a sequence of single independent tasks without taking into account the associations that may occur between them. Such approach leads to the classical concept of recognition algorithm, which assigns a pattern at the $n$-th instant to a class on the base of its features only, namely:

$$
i_{n}=\Psi\left(\mathcal{S}, x_{n}\right), n=1,2, \ldots, i_{n} \in \mathcal{M} .
$$

Thus it will be obtained assuming $a=x_{n}$ for the $n$-th instant. Now, rule derivation is performed based on the whole training set $S$ for which neither the division into sequences $S_{k}$ nor element succession in the sequence is pertinent. Resulting procedure is following:

1. Cover the space $\mathcal{X}^{(l)}$ of the individual feature $x^{(l)}(l=1,2, \ldots, d)$ by overlapping fuzzy sets corresponding to the linguistic "values" of this feature (e.g. small, medium, big, etc.). For each fuzzy set define its membership function. 
Obtained fuzzy sets state premises $A_{i, k}$ in fuzzy rules (5). For example, in the further practical medical diagnosis task, we used triangular fuzzy numbers with 3 regular partitions 6 .

2. For each example generate fuzzy rule with premises corresponding to fuzzy regions with the highest membership grade of appropriate feature.

3. Find the rules with the same premises and aggregate them into one rule.

4. Determine the discrete fuzzy conclusion of the rule (fuzzy set), for which

$$
\mu_{B_{k}}(j)=\frac{n_{k}(j)}{\sum_{j} n_{k}(j)}, j \in \mathcal{M},
$$

where $n_{k}(j)$ denotes the number of learning patterns from $j$-th class fulfilling the $k$-th rule.

Algorithm with k-th Order Context (Mamdani-k). This algorithm makes allowance for the $k$-instant-backwards dependence using full bulk of the measurement data. In effect, we have now $a=\left(x_{n}, x_{n-1}, \cdots, x_{n-k}\right)$ and rule derivation is achieved based on the whole training set $S$, taking into account the succession of particular $k$ elements in sequences $S_{i}$.

\subsection{Algorithms Using Fuzzy Relations}

Algorithm Without Context (Relation-0). This algorithm, as algorithm Mamdani-0, includes no inter-state dependences on a state but it utilizes only the current feature values instead. Application of fuzzy relation to the construction of classifier (6) from the learning set (2) containing $N \times L$ patterns (now the order of patterns in the sequences (3) is irrelevant) is well known in literature [10], [12, 13] and resulting procedure comprises the following items:

1. Cover the space $\mathcal{X}^{(l)}$ of the individual feature $x^{(l)}(l=1,2, \ldots, d)$ by overlapping fuzzy sets corresponding to the linguistic "values" of this feature (e.g. small, medium, big, etc.). For each fuzzy set define its membership function. Obtained fuzzy sets state fuzzified feature space $\mathcal{X}_{F}^{(l)}$ of individual features. Create fuzzified feature space as a product $\mathcal{X}_{F}=\mathcal{X}_{F}^{(1)} \times \mathcal{X}_{F}^{(2)} \times \ldots \times \mathcal{X}_{F}^{(d)}$. Let its cardinality be equal to $d_{F}$ - this value depends on number of partitions and the size of feature vector. For example, in the further practical medical diagnosis task, $d=3$ and we used triangular fuzzy numbers with 3 regular partitions, which gave $d_{F}=27$.

2. Determine observation matrix $O(\mathcal{S})$ of learning set $\mathcal{S}$, i.e. fuzzy relation defined on product of fuzzified feature space $\mathcal{X}_{F}$ and learning set $\mathcal{S}$. The $i$ th row of $O(\mathcal{S})(i=1,2, \ldots, N \times L)$ contains membership degrees of features of $i$ th learning pattern to fuzzy sets of space $\mathcal{X}_{F}$. The number of columns of $O(\mathcal{S})$ is equal to $d_{F}$.

3. Determine decision matrix $D(\mathcal{S})$, i.e. relation defined on product of learning set $\mathcal{S}$ and the set of decisions (classes) $\mathcal{M}$. For the training data, where the classification is exactly known, the $i$ th row is a fuzzy singleton set, i.e. a 
vector of all zeros except for a one at the place corresponding to the class number of $i$ th learning pattern.

4. Find matrix $E(\mathcal{S})$ as a solution of so-called fuzzy relational equation ([1], [13]):

$$
O(\mathcal{S}) \circ E(\mathcal{S})=D(\mathcal{S})
$$

or - in approximate way - as a solution of the following optimization problem:

$$
\rho(O(\mathcal{S}) \circ E(\mathcal{S}), D(\mathcal{S}))=\min _{E} \rho(O(\mathcal{S}) \circ E, D(\mathcal{S})),
$$

where criterion $\rho(A, B)$ evaluates difference between matrices $A$ and $B$, i.e. $\rho(A, B) \geq 0$ and $\rho(A, B)=0$ iff $A=B$. Operator $\circ$ denotes here maxmin-norm composition of relations, i.e. multiplication of matrices $O$ and $E$ with $\times$ and + operators replaced by min and max operators (more general by $t$-norm and $s$-norm operators)([1]). In the further practical example we decided to select the method of determination of matrix $E$, adopting

$$
\rho(A, B)=\sum_{i, j}\left(a_{i j}-b_{i j}\right)^{2}
$$

and applying as an optimization procedure real-coded genetic algorithm.

Matrix $E(\mathcal{S})$ is a fuzzy relation defined on product of decision set $\mathcal{M}$ and feature space $\mathcal{X}_{F}$, in which reflects knowledge contained in the learning set. To classify a new pattern $x$, first the row-matrix of fuzzy observation $O(x)$ is calculated from known vector of its features $\left[x^{(1)}, x^{(2)}, \ldots, x^{(d)}\right]$. Then matrix $E(\mathcal{S})$ is applied to compute an output row-matrix called target vector ([14]):

$$
O(x) \circ E(\mathcal{S})=T(x)=\left[t_{1}(x), t_{2}(x), \ldots, t_{M}(x)\right],
$$

which gives a fuzzy classification in terms of membership degrees $t_{i}(x)$ of the pattern $x$ to the given classes $i=1,2, \ldots, m$. When a crisp decision is required, defuzzification has to be applied, typically according to the maximum rule.

Algorithm with k-th Order Context (Relation-k). This algorithm includes $k$-instant-backwards-dependence $(k<L)$ with full measurement data, i.e. the decision at the $n$-th instant is made on the base of vector of features:

$$
\bar{x}_{n}^{(k)}=\left(x_{n-k}, x_{n-k+1}, \ldots, x_{n-1}, x_{n}\right) .
$$

Although, the main concept of the proposed methods of SC is the same as for independent patterns, there are many differences concerning details in procedure of construction of matrix $E$ and course of recognition process.

Before we will describe these algorithms let us first introduce set $\mathcal{S}^{(k)}$ containing sequences of $(k+1)$ learning patterns from $\mathcal{S}$ and set $\mathcal{S}_{\bar{j}^{(k)}}^{(k)}$ - as previously but in which at the first $k$ position additionally the sequence of classes $\bar{j}^{(k)} \in \mathcal{M}^{k}$ appears. Consequently, the algorithm with $k$-th order dependence (Relation $k$ ) and full measurement features can be presented according to the following points: 
1. Create the fuzzified feature space $\mathcal{X}_{F}$ as in the procedure for independent patterns

2. Determine observation matrix $O^{(k)}$, i.e. fuzzy relation in the space $\mathcal{X}_{F}^{k}=$ $\mathcal{X}_{F} \times \mathcal{X}_{F} \times \cdots \times \mathcal{X}_{F}(k$ times $)$ and learning subset $\mathcal{S}^{(k)}$. The $i$ th row of observation matrix contains memberships degrees of features $\bar{x}^{(k)}$ of $i$ th learning sequence from $\mathcal{S}^{(k)}$ to the fuzzy sets of space $\mathcal{X}_{F}^{k}$.

3. Determine decision matrix $D^{(k)}$, i.e. relation defined on product of learning sequences $\mathcal{S}^{(k)}$ and the set of decisions (classes) $\mathcal{M}$. The $i$ th row of $D^{(k)}$ is a vector of all zeros except for a one at the place corresponding to the last class number of $i$ th sequence in the set $\mathcal{S}^{(k)}$.

4. Find matrix $E^{(k)}$, so as to minimize criterion

$$
\rho\left(O^{(k)} \circ E^{(k)}, D^{(k)}\right) .
$$

Next, at the $n$th step of sequential recognition first the row-matrix of fuzzy observation $O\left(\bar{x}_{n}^{(k)}\right)$ is calculated from known sequence of feature observations (12). Then matrix $E^{(k)}$ is applied to compute a target vector of soft decisions:

$$
O\left(\bar{x}_{n}^{(k)}\right) \circ E^{(k)}=T\left(\bar{x}_{n}^{(k)}\right),
$$

and final crisp decision is obtained after defuzzification step.

\section{Algorithms of SC Based on Rough Sets Theory}

In this section we will apply the rough sets theory 9 to the construction of SC algorithm (4).

Now, the training set (2) is considered as an information system $S=(U, A)$, where $U$ and $A$, are finite sets called universe and the set of attributes, respectively. For every attribute $a \in A$ we determine its set of possible values $V_{a}$, called domain of $a$. Such information system can be represented as a table, in which every row represents a single sequence (3). In successive column of $k$-th row of this table we have values of the following attributes:

$$
x_{1, k}^{(1)}, x_{1, k}^{(2)}, \ldots, x_{1, k}^{(d)}, j_{1, k}, x_{2, k}^{(1)}, x_{2, k}^{(2)}, \ldots, x_{2, k}^{(d)}, j_{2, k}, \ldots, x_{L, k}^{(1)}, x_{L, k}^{(2)}, \ldots, x_{L, k}^{(d)}, j_{L, k} .
$$

In such an information system we can define in different way the subset $C \subseteq A$ of condition attributes and the single-element set $M \subseteq A$ which will be the decision attribute. Consequently, we obtain the decision system $S=(U, C, M)$ in which, knowing the values of condition attributes, our task is to find the value of decision attribute, i.e. to find appropriate pattern recognition algorithm of sequential classification. Of course, as in algorithms based on fuzzy sets theory, we can choose the subset of condition attributes in different way. Taking into account the set of condition attributes $C$, let us denote by $X_{j}$ the subset of $U$ for which the decision attribute is equal to $j, j=1, \ldots, m$. Then, for every $j$ we can defined respectively the $C$-lower approximation $C_{*}\left(X_{j}\right)$ and the $C$-upper approximation $C^{*}\left(X_{j}\right)$ of set $X_{j}[9]$, 17. Hence, the lower approximation of set 
$X_{j}$ is the set of objects $x \in U$, for which knowing values of condition attributes $C$, for sure we can say that they are belonging to the set $X_{j}$. Moreover, the upper approximation of set $X_{j}$ is the set of objects $x \in U$, for which knowing values of condition attributes $C$, for sure we can not say that they are not belonging to the set $X_{j}$. Consequently, we can define $C$-boundary region of $X_{j}$ as follows:

$$
C N_{B}\left(X_{j}\right)=C^{*}\left(X_{j}\right)-C_{*}\left(X_{j}\right)
$$

For every decision system we can formulate its equivalent description in the form of set of decision formulas $\operatorname{For}(C)$. Each row of the decision table will be represented by single if-then formula, where on the left side of this implication we have logical product (and) of all expressions from $C$ such that every attribute is equal to its value. On its right side we have expression that decision attribute is equal to the one number of class from (11). These formulas are necessary for constructing different pattern recognition algorithms for sequential classification.

\subsection{Algorithm Without Context (Rough-0)}

As usual, we start with the algorithm without the context which is well known in literature $([3,5], 9], 10])$. In this case our decision table contains $N \times L$ patterns, each having $d$ condition attributes (features) and one decision attribute (the class to which the pattern belongs).

Application of rough set theory to the construction of classifier (6) from the learning set (2) can be presented according to the following items:

1. If the attributes are the real numbers then the discretization preprocessing is needed first. After this step, the value of each attribute is represented by the number of interval in which this attribute is included. Of course for different attributes we can choose the different numbers of intervals in order to obtain their proper covering and let us denote for $l$-th attribute $(l=1, \ldots, d)$ by $\nu_{p}^{l} l$ its $p_{l}$-th value or interval.

2. The next step consists in finding the set $\operatorname{For}(C)$ of all decision formulas from (2), which have the following form:

$$
I F\left(x^{(1)}=\nu_{p 1}^{l}\right) A N D \ldots A N D\left(x^{(d)}=\nu_{p d}^{d}\right) \operatorname{THEN\Psi }(\mathcal{S}, x)=j .
$$

Of course, it can happen that from the learning set (2) we obtain more than one rule for particular case. Then for such a formula (17) we determine its strength factor [5], which is the number of correctly classified patterns during learning procedure. If any case in (2) is single then the strength factor of corresponding rule is equal to one.

3. For the set of formulas $\operatorname{For}(C)$, for every $j=1, \ldots, m$ we calculate their $C$-lower approximation $C_{*}\left(X_{j}\right)$ and their boundary regions $C N_{B}\left(X_{j}\right)$.

4. In order to classify $x_{n}$ (after discretization its attributes if it is necessary) we look for matching rules in the set $\operatorname{For}(C)$, i.e. we take into account such rules in which the left condition is fulfilled by the attributes of recognized pattern. 
5. If there is only one matching rule, then we classify this pattern to the class which is indicated by its decision attribute $j$, because for sure such rule is belonging to the lower approximation of all rules indicating $j$, i.e. this rule is certain.

6. If there is more then one matching rule in the set $\operatorname{For}(C)$, it means that the recognized pattern should be classified by the rules from the boundary regions $C N_{B}\left(X_{j}\right), j=1, \ldots, m$ and in this case as a decision we take the index of boundary region for which the strength of corresponding rule is maximal. In such a case we take into account the rules which are possible.

\subsection{Algorithm with $k$-th Order Context (Rough-k)}

Although as in [17], we could take into account at $n$-th instant whole available information about the state of recognized sequential process, for the same reason as previously let us choose the following decision atributes in our decision table:

$$
x_{n-k}^{(1)}, \ldots, x_{n-k}^{(d)}, x_{n-k+1}^{(1)}, \ldots, x_{n-k+1}^{(d)}, \ldots, x_{n-1}^{(1)}, \ldots, x_{n-1}^{(d)}, \ldots, x_{n}^{(1)}, \ldots, x_{n}^{(d)} .
$$

This means that algorithm includes $k$-instant-backwards-dependence $(k<L)$ with full measurement data. Let us denote by $D$ the total number of decision attributes (former was $D=d$ and now $D=(k+1) \times d+k)$. Next, from the (2), we can create the decision table which will have $D+1$ column (the last one is the true classification of $n$-th recognized pattern) and consequently the number of rows will be equal to $N \times(L-k)$, because from each sequence (3) we can obtain $L-k$ subsequences of the length $k+1$. The main idea of the proposed methods of SC is the same as for independent patterns but there are differences concerning details in procedure of construction of the set of decision formulas $\operatorname{For}(C)$. Now, the decision formulas are of the following form:

$$
I F\left(x^{(1)}=\nu_{p 1}^{l}\right) A N D \ldots A N D\left(x^{(D)}=\nu_{p D}^{D}\right) \operatorname{THEN} \Psi\left(\mathcal{S}, \bar{x}_{n}^{(k)}\right)=j_{n}
$$

The next steps of SC are the same as previously, i.e. we calculate $C_{*}\left(X_{j_{n}}\right)$ and $C N_{B}\left(X_{j_{n}}\right)$ and finally, the decision is made according to same procedure.

All the decision algorithms that are depicted in the previous sections have been experimentally tested in respect of the decision quality (frequency of correct classifications) for real data that concern recognition of human acid-base equilibrium states (ABE).Results of experimental investigations are presented in the next section.

\section{Medical Example: Sequential Diagnosis of Acid-Base State Balance}

In the course of many pathological states, there occur anomalies in patient's organism as far as both hydrogen ion and carbon dioxide production and elimination are concerned, which leads to disorders in the acid-base equilibrium (ABE). 
Thus we can distinguish acidosis and alkalosis disorders here. Each of them can be of metabolic or respiratory origin, which leads to the following ABE states classification: metabolic acidosis, respiratory acidosis, metabolic alkalosis, respiratory alkalosis, correct state.

In the process of treatment, correct recognition of these anomalies is indispensable, because the maintenance of the acid-base equilibrium, e.g. the $\mathrm{pH}$ stability of the fluids is the essential condition for correct organism functioning. Moreover, the correction of acid-base anomalies is indispensable for obtaining the desired treatment effects.

In medical practice, only the gasometric examination results are made to establish fast diagnosis, although the symptom set needed for correct ABE estimation is quite large. The utilized results are: the $\mathrm{pH}$ of blood, the pressure of carbon dioxide, the current dioxide concentration.

An anomalous acid-base equilibrium has a dynamic character and its changes depend on the previous state, and in consequence they require frequent examinations in order to estimate the current $\mathrm{ABE}$ state. It is clear now that the $\mathrm{SC}$ methodology presented above suits well the needs of computer aided ABE diagnosing. The current formalization of the medical problem leads to the task of the ABE series recognition, in which the classification basis in the $n$-th moment constitutes the quality feature consisting of three gasometric examinations. And the set of diagnostic results $\mathcal{M}$ is represented by 5 mentioned acid-base equilibrium states.

The diagnostic algorithms applied to the ABE which state sequential diagnosis task have been worked out on the basis of evidence material that was collected in Neurosurgery Clinic of Medical Academy of Wroclaw and constitutes the set of training sequences (2). The material comprises 78 patients (78 sequences) with $\mathrm{ABE}$ disorders caused by intracranial pathological states for whom the gasometric examination results and the correct $\mathrm{ABE}$ state diagnosis were regularly put down on the 12-hour basis. There were around 20 examination cycles for each patient, yielding the total of 1416 single examination instances.

To compare the classification accuracy of proposed concepts of SC algorithms and the performance of RGA, ten independent runs of RGA were carried out for each diagnostic algorithm with different random initial populations. The results are shown in Table 1. The values depicted in the table are those of the best solution obtained at the end of a RGA trial. Table 1 contains also the best result, the mean value and standard deviation for each SC algorithm. In testing of Mamdani inference system and algorithms based on rough sets theory, the cross validation method was used, i.e. for every trial ten testing sequences were chosen randomly.

These results imply the following conclusions:

1. Algorithms Mamdani-0, Relation-0 and Rough-0 that do not include the inter-state dependencies and treat the sequence of states as independent objects are worse than those that have been purposefully designed for the sequential medical diagnosis task, even for the least effective selection of input data. This confirms the effectiveness and usefulness of the conceptions and 
Table 1. Frequency of correct diagnosis for various diagnostic algorithms (in per cent)

\begin{tabular}{llllllllll}
\hline Trial & Ma-0 & Ma-1 & Ma-2 & Re-0 & Re-1 & Re-2 & Ro-0 & Ro-1 & Ro-2 \\
\hline 1 & 66.9 & 72.1 & 67.3 & 80.6 & 89.6 & 91.8 & 83.5 & 85.7 & 90.1 \\
2 & 68.1 & 69.2 & 70.2 & 82.2 & 86.5 & 91.9 & 85.1 & 86.1 & 89.6 \\
3 & 67.5 & 70.1 & 70.1 & 79.4 & 87.2 & 88.9 & 83.8 & 88.0 & 91.1 \\
4 & 68.0 & 71.2 & 71.6 & 78.5 & 85.9 & 92.6 & 84.0 & 88.5 & 92.0 \\
5 & 67.8 & 69.1 & 71.2 & 80.9 & 90.3 & 91.9 & 83.6 & 87.6 & 89.8 \\
6 & 67.9 & 70.0 & 69.4 & 82.1 & 89.7 & 91.6 & 85.7 & 86.4 & 89.9 \\
7 & 67.2 & 71.2 & 70.9 & 81.9 & 88.1 & 89.4 & 83.1 & 85.1 & 90.2 \\
8 & 68.7 & 69.8 & 71.2 & 78.3 & 87.2 & 89.0 & 85.9 & 86.9 & 91.4 \\
9 & 67.1 & 68.6 & 70.4 & 78.5 & 90.7 & 92.9 & 83.4 & 88.9 & 91.3 \\
10 & 67.0 & 68.2 & 67.6 & 81.1 & 88.7 & 92.8 & 86.9 & 87.8 & 91.2 \\
\hline Best & 68.7 & 72.1 & 71.6 & 82.2 & 90.7 & 92.9 & 84.0 & 88.9 & 92.0 \\
Mean & 67.6 & 70.0 & 70.0 & 80.3 & 88.4 & 91.3 & 84.5 & 87.1 & 90.7 \\
\hline
\end{tabular}

algorithm construction principles presented above for the needs of sequential diagnosis.

2. There occurs a common effect within each algorithm group: the model of the second order dependency (Mamdani-2, Relation-2, Rough-2) turns out to be more effective than the first order dependence approach (Mamdani-1, Relation-1, Rough-1).

3. There is no essential difference among the algorithms using the same input data which are based either on fuzzy relation method or on rough sets theory.

4. The RGA method is capable of solving the problem of learning of SC algorithm for practical computer-aided medical diagnostic system. Results of RGA performances turn out to be quite repeatable and insensitive to initial conditions.

It must be emphasized that proposed procedures leads to the very flexible sequential recognition algorithm due to optional value of $k$. In particular the value of $k$ need not be constant but it may dynamically change from step to step. So, the choice $k=n-1$ for $n$-th instant of sequential classification denotes the utilization of the whole available information according to the general form of decision rule (4). On the other hand however, such a concept - especially for bigger $n$ - is rather difficult for practical realization.

\section{Conclusions}

The aim of this work was comparative analysis of soft computing methods applied to the sequential classification tasks in which there exist dependencies among the patterns to be recognized. Two approaches to SC task were considered: using fuzzy sets theory and using rough sets theory. For both of them corresponding algorithms were proposed, without taking into account the treatment directly (of course results of treatment are observed indirectly in feature 
vectors). The empirical results show that in such case the accuracy of classification can be improved taking into account such dependencies however, more empirical studies are required. Moreover, in presented practical example there is no essential difference among the results of algorithms using the same input data which are based either on fuzzy sets or on rough sets theory. It means, that such soft computing methods in SC task can be considered as complementary.

\section{References}

1. Czogala, E., Leski, J.: Fuzzy and neuro-fuzzy intelligent systems. Springer, New York (2000)

2. Dinola, A., Pedrycz, W., Sessa, S.: Fuzzy relation equations theory as a basis of fuzzy modelling: an overview. Fuzzy Sets and Systems 40, 415-429 (1991)

3. Fang, J., Grzymala-Busse, J.: Leukemia Prediction from Gene Expression Data-a Rough Set Approach. In: Rutkowski, L., Tadeusiewicz, R., Zadeh, L., Zurada, J. (eds.) Artificial Intelligence and Soft Computing, pp. 899-908. Springer, New York (2006)

4. Goldberg, D.: Genetic algorithms in search, optimization and machine learning. Addison-Wesley, New York (1989)

5. Grzymala-Busse, J.: A System for Learning from Examples Based on Rough Sets. In: Slowinski, R. (ed.) Intelligent Decision Support: Handbook of Applications and Advances of the Rough Sets Theory, pp. 3-18. Kluwer Academic Publishers, Dordrecht (1992)

6. Kurzynski, M.: Multistage diagnosis of myocardial infraction using a fuzzy relation. LNCS (LNAI), pp. 1014-1019. Springer, New York (2004)

7. Kurzynski, M., Zolnierek, A.: Sequential Classification via Fuzzy Relations. In: Rutkowski, L., Tadeusiewicz, R., Zadeh, L., Zurada, J. (eds.) Artificial Intelligence and Soft Computing, pp. 623-632. Springer, New York (2006)

8. Michalewicz, Z.: Genetic Algorithms + Data Structure = Evolution Programs Springer, New York (1996)

9. Pawlak, Z.: Rough Sets - Theoretical Aspect of Reasoning About Data. Kluwer Academic Publishers, Dordrecht (1991)

10. Pawlak, Z.: Rough Sets, Decision Algorithms and Bayes' Theorem. European Journal of Operational Research 136, 181-189 (2002)

11. Pedrycz, W.: Fuzzy Sets in Pattern Recognition: Methodology and Methods. Pattern Recognition 23, 121-146 (1990)

12. Pedrycz, W.: Genetic Algorithms for Learning in Fuzzy Relation Structures. Fuzzy Sets and Systems 69, 37-45 (1995)

13. Ray, K., Dinda, T.: Pattern classification using fuzzy relational calculus. IEEE Transactions on Systems, Man and Cybernetics 33(1), 1-16 (2003)

14. Setnes, M., Babuska, R.: Fuzzy relational classifier trained by fuzzy clustering. IEEE Transactions on Systems, Man and Cybernetics 29, 619-625 (1999)

15. Wang, L.X., Mendel, J.M.: Generating fuzzy rules by learning from examples. IEEE Trans. on Systems, Man and Cybernetics 22, 1414-1427 (1992)

16. Wang, L.X.: A course in fuzzy systems and control. Prentice-Hall, New York (1998)

17. Zolnierek, A.: Application of rough sets theory to the sequential diagnosis. In: Maglaveras, N., Chouvarda, I., Koutkias, V., Brause, R. (eds.) ISBMDA 2006. LNCS (LNBI), vol. 4345, pp. 413-422. Springer, Heidelberg (2006) 